\title{
Data Processing for Scanning Tunneling Spectroscopy Analysis of Metal and Semiconductor Areas in Nanoscale
}

\author{
T.M. Nenchuk, P.V. Galiy, Ya.M. Buzhuk, K.A. Korvats'ka \\ Electronics and Computer Technology Department \\ Ivan Franko Lviv National University \\ Lviv, Ukraine \\ nenchuk@electronics.Inu.edu.ua
}

\begin{abstract}
Algorithm for sequential actions and calculations is proposed, which yields the current-voltage curves (CVC) from the matrix of experimental data acquired by scanning tunneling spectroscopy analysis of metal-semiconductor planar systems. CVCs' study in the vicinity of a zero-bias voltage range that corresponds to the width of the band gap of a semiconductor allows to expand the capabilities of existing software data processing systems and to increase the planar resolution and accuracy of determining the relative concentrations of metal and semiconductor on a nanoscale by means of a scanning tunneling microscope. To implement the analysis algorithm, a program has been developed with a user-friendly interface and the ability to visualize the data and to calculate the relative concentrations of the metal and semiconductor.
\end{abstract}

Index Terms - Data science; data formats; data processing for scanning tunneling spectroscopy; applied software.

\section{INTRODUCTION}

The processing and analysis of data obtained for solids surfaces by the scanning tunneling spectroscopy (STS) method for a group of researchers working remotely is a rather topical problem, because today the software of scanning probe microscopes (SPM), as a rule, uses its own formats for the preservation of experimental data. For example, Omicron NanoTechnology STM / AFM System stores information in a file with spectral recording parameters sequentially in the text format as a header, and a pixel matrix data for the scanned surface area in binary formats. Consequently, there is a problem of converting data formats in the process of transition from data acquisition to their processing in order to obtain reliable conclusions about the results of scanning probe analysis.

\section{BACKGROUD OWERVIEW}

At present, free software products such as Gwyddion and WSxM [1, 2] are used to process data of SPM, besides its own software of experimental equipment. Gwyddion is a modular program for SPM data visualization and analysis. In the first place, it is designed to analyze the surface topography and supports many SPM data formats. However, at present, it does not have the functionality to analyze the spectrum of the STS.
The later versions of WSxM allow raw data processing by STS and used by us during the investigation of the STS spectra of the metal semiconductor systems to read and convert information from the output binary code into a user-friendly data format, e.g., $x s l x$.

Visual representation of raw STS data by the last mentioned software is limited to various kinds of representations, such as differentiation and normalization of data, data acquisition for local points, and integration for a selected array on the surface. However, for specific case analysis, it is necessary to develop special analysis algorithms based on proper information from a specific subject area.

Obviously, for STS studies, the current-imaging tunneling spectroscopy (CITS) spectroscopy mode is used, which provides record of current-voltage curves (CVC) for the pixels of the topographic or, so-called, scanning tunneling spectroscopy (STM) image [3]. Using these curves allows user to estimate the local electron structure of the surface, since the dependence of $\mathrm{dI} / \mathrm{dV}$ on the bias $\mathrm{V}-(\mathrm{dI}(\mathrm{V}) / \mathrm{dV}=\mathrm{f}(\mathrm{V}))$ is proportional to the local density of the surface states. Thus, CITS mode combines the analysis by the STS method with the construction of appropriate STM images.

As a result of the CITS methodology in the Omicron data format, 110-300 spatial maps of tunneling current are obtained at certain bias voltages of the SPM tip - the surface of the crystal in the range from $-\mathrm{U}_{\text {bias }}$ to $+\mathrm{U}_{\text {bias, }}$, which are interpreted as the reflection of the relative magnitude of the local density of surface states (LDOS) that participate in tunneling processes.

\section{RESULTS AND DISCUSSION}

On the basis of these data, the WSxM provides an opportunity to represent the CVC of the tip - the surface of the sample system as averaged over the selected area, and for the selected points on the surface, or alternatively, all points of the analysis. By default, the number of analysis points of the spectrum recorded in the STS file is 6400 (a matrix of $80 \times 80$, regardless of the size of the scanned area in $\left.\mathrm{nm}^{2}\right)$. This is substantially less than the matrix of the results of scanning the area of the same size by the STM method (matrix 400x400). 
Therefore, simultaneously received STM images during STS scanning have significantly less resolution, which impairs the possibility of comparing topographical specialties and their CVC.

We propose a method for detailed flow analysis of an array of CVC obtained for a selected area on a sample surface. The conversion of the STS CITS spectrum to the format available for reading by spreadsheets is implemented using the WSxM program, which allows you to write CVC data in the text format. The resulting data array of size $\mathrm{U}_{\mathrm{N}} \times 12800$ presents a pair of columns of bias voltage values sampled with some step $\Delta \mathrm{U}$ (in the range $-\mathrm{U}_{\text {bias }}-$ to $+\mathrm{U}_{\text {bias }}$ ) and, corresponding to them, the local tunneling current. For example, typical values for $\mathrm{N}$ are 110 or 300 . In fact, in the process of STS data analysis, we deal with a three-dimensional data structure, where each pair of the columns bias voltage and tunneling current is tied to a specific local point of analysis on the studied surface. The discreteness of these points in the nanoscale along the successive scans on the surface depends on the size of the analyzed region selected for scanning at a standard number of readings, as mentioned above. Based on the limitations of the experimental data collection method the scanned area of $10 \mathrm{x}$ $10 \mathrm{~nm}^{2}$ in size is sufficient to get reliable conclusions. That is, the corresponding pairwise values of the bias voltage - the tunneling current vectors are obtained with a discreteness of $1.25 \AA$. This is quite enough to cover the entire area of the analysis, while it is known that the resolution of the method for obtaining experimental data is in the vicinity of $1 \AA$.

The technical result is achieved by the fact that an algorithm for sequential actions and calculations is obtained, which yields the CVCs from the matrix of experimental data in the vicinity of the range of zero bias voltage corresponding to the width of the bandgap of the semiconductor. The absence of a tunneling current in the vicinity of the zero-offset from the voltage range, which makes it possible to trace the energy levels between the top of the valence band $\mathrm{E}_{\mathrm{v}}$ and the bottom of the conduction band $\mathrm{E}_{\mathrm{c}}$, due to the absence of a local density of electronic states in the semiconductor bandgap, which could participate in the tunneling process: for negative bias - in the direction from sample to the tip and vice versa for positive bias voltage. The use of STS to determine the presence of metallic nanostructures and their placement on the material surface is based on a substantially different behavior of the currentvoltage characteristics at atomic resolution points obtained for the metal - linear CVC and for semiconductors - nonlinear $\mathrm{CVC}$ in the range of biases stresses that are responsible for probing of semiconductor gap [4]. Figs. 1, 2 show typical CVCs of tunnel contact in the case of scanning the surface of the semiconductor and metal, consequently.

Testing the analysis algorithm was applied using raw data obtained by Omicron Nano Technology STM / AFM System, which provides ultra-high vacuum STS analysis at a pressure of residual gases less than $10^{-10}$ Torr at room temperature. For example, was selected layered semiconductor crystals InSe intercalated by nickel (Ni). Such crystals are convenient model objects to study systems with low dimensionality. One of the defining features of layered semiconductor crystals, in particular, InSe, is the presence of interlayer van der Waals gap, allowing intercalation that means introducing impurity atoms such as 3d-atoms of iron group including nickel, chromium and others. Because layered crystals are easily cleaved along van der Waals interlayer plane, application of STM / STS techniques, which are research methods for solid surface, gives possibility to study the bulk of crystal layers that due to cleavage come out on the (0001) surface.

Fig. 3 shows an array of CVCs obtained for the system tungsten tip - the surface (0001) $\mathrm{Ni}_{\mathrm{x}} \mathrm{InSe}$, obtained in CITS mode for an array of 6400 study points spatially distributed on a surface area with a size of $10 \times 10 \mathrm{~nm}^{2}$. Circles on the curves indicate the corresponding discrete experimental values obtained from the sampling step at the bias voltage, which is equal to $0.035 \mathrm{~V}$.

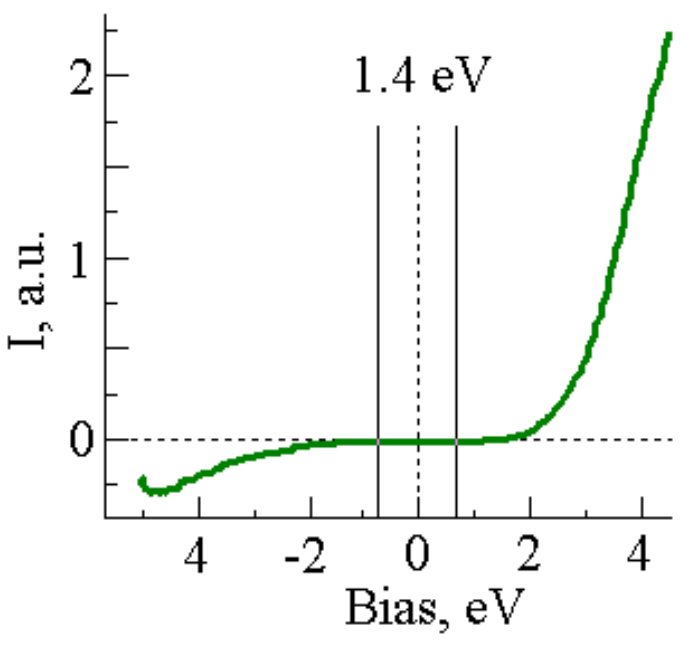

Figure 1. CVC of tunnel contact tip-semiconductor. Vertical lines show boundaries of energy gap in semiconductor.

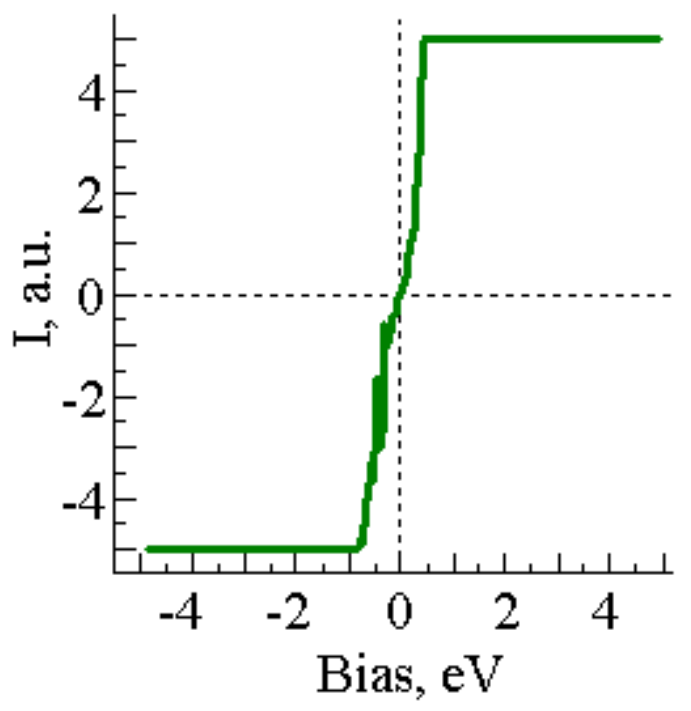

Figure 2. CVC of tunnel contact tip-metal cluster on the surface.

The presence of intercalated metal on the surface can be easily determined by the presence of a tunnel current with a value greater than a certain background level which is recorded in the range of bias voltage, which allows probing the width of 
the InSe band gap $(1.4 \mathrm{eV})$. The calculation of the concentration of nickel on the surface, carried out according to the proposed method for small samples of STS data from different areas of the surface of $10 \times 10 \mathrm{~nm}^{2}$, gives an average assessment of the metal concentration at $1 \%$, varying within $0,8-1,25 \%$ for different areas.

The reliability of determining the relative concentrations of metal on the surface of the sample depends on the thickness of the metal layer. In the case when the dispersed metal phase does not exceed the thickness of the monolayer, the above method of concentration determining is suitable for use, and the results are reliable, otherwise, additional studies are necessary.

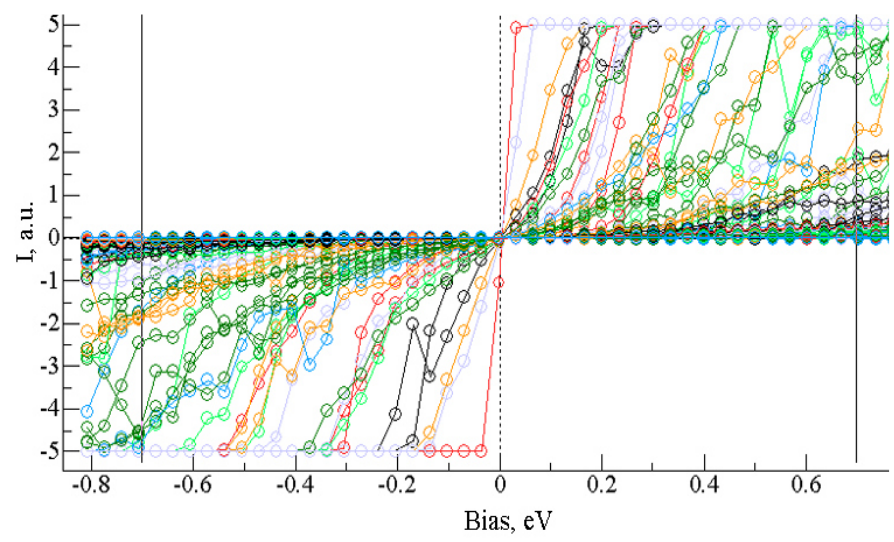

Figure 3. An array of 6400 local CVCs obtained as a result of a scan of an area measuring $10.2 \times 10.2 \mathrm{~nm}^{2}$ on the surface of a $\mathrm{Ni}_{\mathrm{x}} \mathrm{InSe}$ metalsemiconductor hybrid system in the range of bias voltage, which is restricted to the InSe-gap $(1.4 \mathrm{eV})$. The circles on the curves depict discrete data from the experimental data matrix.

For the crystalline structure of a layered InSe crystal, the interlayer distance does not exceed $3.08 \AA$ [5], which is commensurate with the doubly atomic radius of nickel. Therefore, it can be argued that nickel nano clusters are distributed on a surface with a thickness not exceeding a monolayer. The presented results of determining the relative concentration of metal and semiconductor on the surface are reliable, which confirms the obtaining of the expected technical result.

To implement the proposed algorithm of analysis, a program on $\mathrm{C} \#$ has been developed.

The main functional requirements for its development were the next. The program should read the data from the $x l s x$ file of the specified format. The user must get an interface where you can press the button through the dialog to select the input file. Next, the program reads the necessary data and processes them in an array, which will be used during the execution of the program. The user should be able to view the CVC characteristics based on several parameters such as minimum and maximum voltage and the choice of a point analysis to display the current value at the output. The construction of a two-dimensional surface with the indication of the threshold value of the current value, above which, it is believed, the metal is present at a given point.

Also, the user must be able to select a specific point on the surface image, after which a three-dimensional model of the selected point and adjacent points will open, where the pixel color will correspond to the value of the current value at each point.

Fig.4 show dialog window of user interface after reading matrix of experimental data for selected area of analysis on the surface of $\mathrm{Ni}_{\mathrm{x}} \mathrm{InSe}$ system.

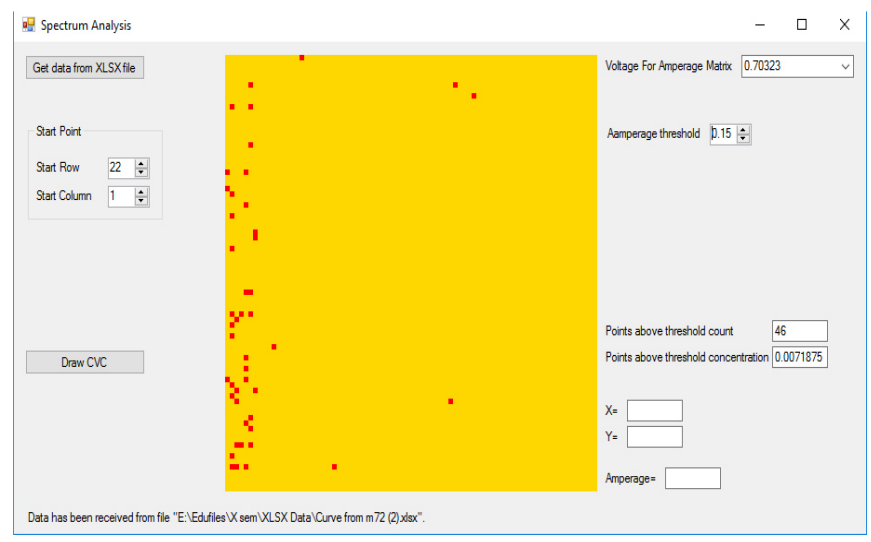

Figure 4. User interface with spatial visualization of metal inclusions on the investigated area of the semiconductor surface and the quantitative value of the relative concentration of metal on the surface of the semiconductor.

Its main features are the next:

since the raw data file format may have different address locations for analyzing cells, the interface has the ability to select the address of the source cell to start reading the data into a working array;

works correctly with a different dimension of the raw data matrix, which is due to the step of sampling over the bias voltage;

the choice of the tunnel current level above which point of analysis can be attributed to the class of metallicity is provided by option Amperage threshold;

allows you to set the range of analysis of data series, which is limited by the bias voltage, which correlates with the width of the semiconductor bandgap;

displays the results of calculation of the absolute quantity of points on the analysis surface that meet the user selected criteria and the relative concentration of the metal and the semiconductor;

allows to display CVC that corresponds to point on the twodimensional image selected by pointer;

builds a three-dimensional picture in a color gamut, which depends on the value of the tunneling current of $5 \times 5$ neighboring points from the one specified by user. 


\section{CONCLUSIONS}

The algorithm of frequency analysis for the matrix of raw data obtained by STS experimental setup is proposed for the purpose of obtaining the degree of semiconductor surface metallization with the calculation of relative metal and semiconductor concentrations on nanoscale.

The basis of this approach lies in a substantially different behavior of the CVC for the metal and the semiconductor in the range of the biases probing tip - sample that corresponds to the width of the semiconductor bandgap.

Such an approach is easily formalized and for its implementation, an application has been developed with the help of which it is possible to quickly process a large series of raw experimental data.

\section{REFERENCES}

[1] P. Klapetek, D. Necas, and C. Anderson, "Gwyddion user guide," 2004 2007, 2009-2016: http://gwyddion.net.

[2] I. Horcas, R. Fernandez, J.M. Gomez-Rodriguez, J. Colchero, J. GomezHerrero, and A.M. Baro, "WSXM: a software for scanning probe microscopy and a tool for nanotechnology," Rev. Sci. Instrum., vol. 78, pp. 013705-013713, January 2007.

[3] R.Addou, R.M. Wallace, " Surface Analysis of WSe $\mathrm{W}_{2}$ Crystals: Spatial and Electronic Variability," ACS Appl. Mater. Interfaces, vol. 8, No.39, pp. 26400-26406, October 2016.

[4] S. Asano, H. Fujitani, M. Tsukada, "Theoretical Approaches to MetalSemiconductor Interfaces," in Metal-Semiconductor Interfaces, Akio Hiraki, Ed. Ohmsha, Japan: IOS Press, 1995, pp. 1-23.

[5] Y.Zhirko, V.Trachevsky and Z.Kovalyuk, "On the Possibility of Layered Crystals Application for Solid State Hydrogen Storages - InSe and GaSe Crystals," in Hydrogen Storage, Chapter 9, J. Liu Ed., InTech, 2012, DOI: $10.5772 / 50307$. 